Более общую модель электромагнитного поля можно получить при врацении ротора с переменной скоростью $\omega(\mathrm{t})$.

Таким образом, если на вращағощемся роторе установить несколько электродов, заряженных выпрямленным напряженнем, и, изменяя параметры $\alpha,(0, \mathrm{a}, \mathrm{h}$, можно получить электромалнитное поле заданной формы (дискретное или непрерывное), определенной амплитуды, частоты и фазы

\title{
Литература
}

1 Машек Ч.М., Синицын Е.В., Машек А.Ч. Расчет электрического поля системы технологической транспортировки порошков - Ленинград:Известия Ленинградского электротехнического института им. В.И.У Јьянова, вын.166,1983, с. 104.

2. Н.П. Верецагин, В.И.Левитов, Г.З.Мирзабекян, М.И.Пашин. Основы электрогазодинамики дисперсных систем- М.: Энергия. 1984c. 324.

3. Балога М.К., Литинский Г.А.Электро-антисептирование в пищевой промышленности- Кишинев Штиница, 1988,c. 180

4. Lord P R. Elektrostatic forces in Processing Textile Materials, Textile MFR, V. 88 , nov., 1982

УДК $664.046: 65.011 .46$

\section{СИСТЕМЫ ГАРАНТИРУЮЩЕГО УПРАВЛЕНИЯ ПРОЦЕССАМИ СУШКИ ЗЕРНА}

Хоб́ин В.А., Д-р техн. наук, профессор, Степанов М.Т., канд. техн. наук, доцент Одесская национальная академия пишевых технологий

Рассиатривается прииенение систеи гараитируоцего управления для повьиеии эиергетической эррективности пролессов суики зерна. Алгоритиы паких систем позволяют реиить две новые задачи: I) перераспределение ресурсов .правления иежду температураии зерна и суиильных агентов; 2) ведение проиесса в окрестности грании допустимых и энерго фффективных режииов суиии.

Application of guaranteeing control systems for increase of power efficiency of grain drying processes is considered. Algorithms of such systems allow to solve two new problems. 1) redistribution of resources of management between temperatures of a grain and drying agents, 2) conducting process in a vicinity of borders allowable and power-efficacious drying conditions.

Ключевые слова: системы гарантирующего управления, сушка, зерно, энергетическая эффективность, управление

ІІроцесс сушки зерна гребует значительных энергетических затрат. IІредприятия часто стремятся минимизировать его применение за счет поиска компромисса между снижением прибыли из-за потерь сухой массы и качества зерна при хранении, с одной стороны, и затратами на процесс сушки, с другой. Снижение удельных энергетических затрат на сушку и соблюдение всех требований технологии при реализации процесса является комплексной проблемой и, в общем случае, гребует усилий разработчиков техники, технологии, систем автоматизации. Не оспаривая потенциальных возможностей двух первых направлений, мы считаем, что на данном этапе наиболее значительный экономический эффект может быть получен за счет оснащения зерносушилок современными системами автоматического управления (САУ). ІІоэтому цель исследования повысить зффективность работы шахтных прямоточных зерносушилок, снизив удельные затраты энергии, потери качества зерна и повысив производительность, за счет совершенствования структуры и алгоритмов систем автоматизации. В качестве основы такого совершенствования выбраны принципы, разработанные в классе систем гарантирующего управления (СГУ) и систем с прогнозированием.

Сушилка является сложным объектом для эффективного управления. Эта сложность объясняется следуюшими основными причинами (здесь и далее - на примере зерносушилки ДСІІ-32от):

а) на режим работы зерносушилок технологическим регламентом наложено минимум пять ограничений: на температуры сушильных агентов (СА) и зерна для первой и второй зон и на влажность зерна после сушки. При этом количество управляющих воздействий, реально доступных для воздействия на процесс, только три: изменение расхода топлива, подаваемого в топку, изменение степени подсоса наружного воздуха к СА, подаваемому в первую зону сушки, и изменение производительности сушилки. Очевидно, что при традиционных подходах к управлению гарантировать их одновременное выполнение при изменениях условий сушки невозможно;

б) удельные энергозатраты на сушку будут тем меньше, чем ближе значения режимных параметров будут 
приближаться к своим огранччениям, но нарушения этих ограничений будут приводить к существенным потерям качества зерна. Цля максимально достижимой эффективности процесса сушки необходимо искать компромисс между этими взаимно противоречивыми факторами. Очевидно, что он будет изменяться, и будет зависеть от конкретных условий сушки.

САУ процессом сушки, реализованная, как СІУ, см. [1], позволяет преодолеть описанные выше проблемы и повысить эффективность процесса сушки при строгом соблюдении технологических регламентов. В рамках принципов гарантирования, этой САУ реализуются следующие функции:

а) переключения ресурсов управляющих воздействий с регулирования температуры СА на температуры зерна (и, наоборот) по результатам оценки вероятностей нарушения ими соответствуюших ограничений;

6) регулирования влажности зерна на выходе зерносушилки изменением ее производительности, обеспечивая устойчивость и качество процесса регулирования за счет применения специальных алгоритмов с прогнозированием;

в) оптимизации процесса по критерию минимума удельных энергозатрат за счет ведения его в режимах, максимально близких к ограничениям регламента и, одновременно, при гарантировании их соблюдения с наперед заданной вероятностью.

Структурная схема СГУ для сушилки ДСП-32от приведена на рис. 1

Модули МРЗД рассчитывают допустимые заданные значения для каждой регулируемой переменной (для обшности они обозначены переменной $\bar{y}=\left\{y_{i=1,5}=\left\{T_{c a l}, T_{c a 2}, T_{31}, T_{32}, W_{i x}\right\}^{T}\right.$ :

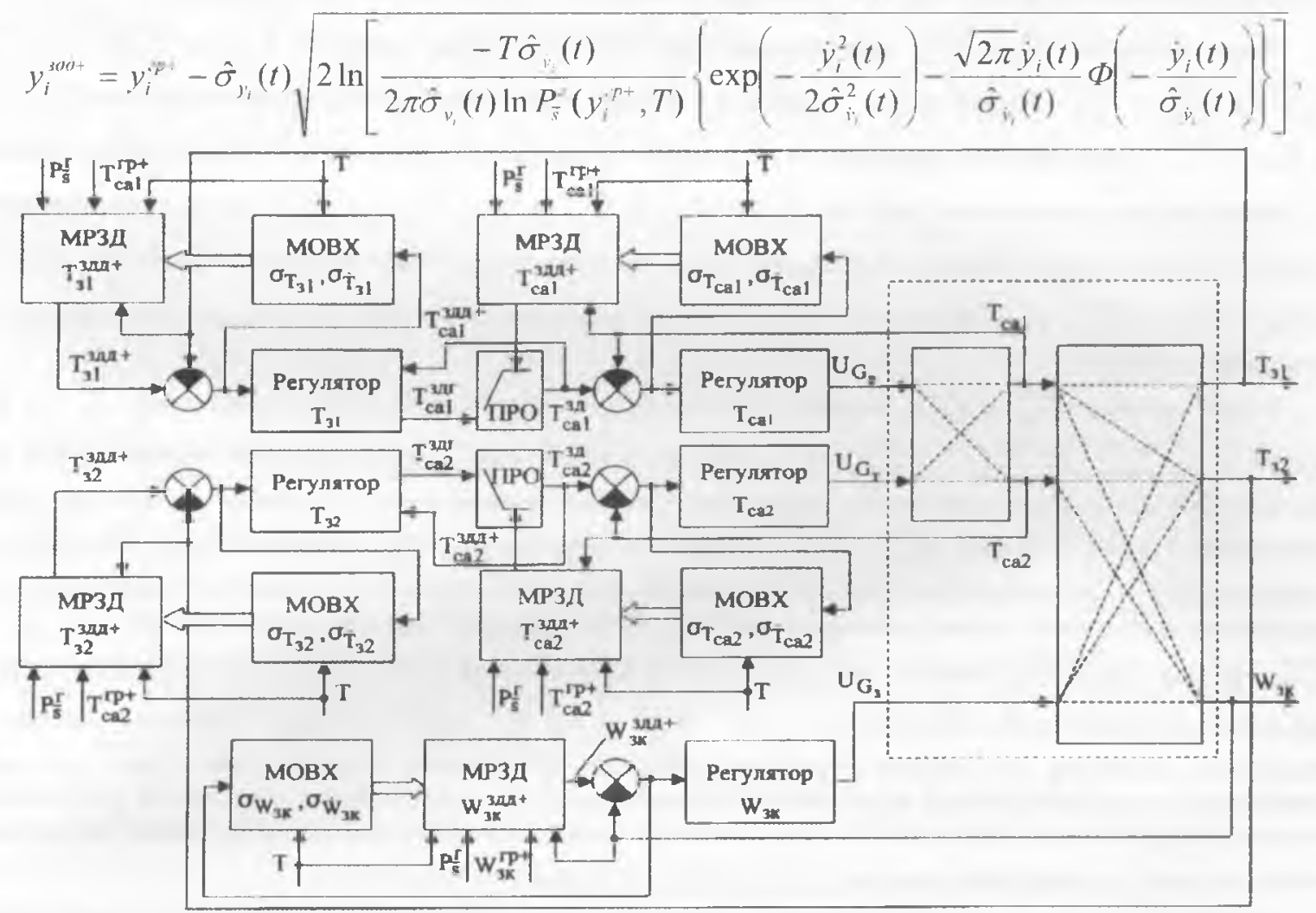

Рис. 1 - Структурная схема СГУ зерносушилкой ДСП-32от (МРЗД - модуль расчета допустимого' заданного значения, МОВХ - модуль оценки вероятностных характеристик, ПГРО - повторители с регулируемым верхним ограничением)

где $y_{1}^{2 p t}-$ значение верхней границы регламента;

$P_{\bar{i}}^{2}\left(y_{i}^{. p+}, T\right)$ - заданная вероятность отсутствия выхода $y_{l}(t)$ за $y_{i}^{2 p^{+}}$;

$T$ - скользящий интервал времени на котором СГУ гарантируется отсутствие выхода $y_{t}(t)$ за $y_{i}^{c p}$ с вероятностью $P_{\bar{s}}^{2}\left(y_{i}^{c p}, T\right)$;

$\hat{m}_{v_{i}}(t)$ и $\hat{m}_{\hat{v}_{i}}(t), \hat{\sigma}_{y_{i}}(t)$ и $\hat{\sigma}_{v_{i}}(t)$ - оценки математических ожиданий и среднеквадратических отклонений, рассчитываемых на скользящих интервалах времени в MOBX:

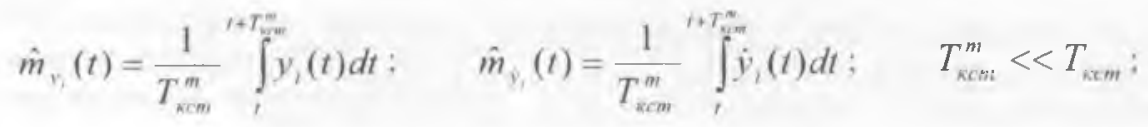




$$
\hat{\sigma}_{v_{i}}(t)=\sqrt{\frac{1}{T_{k c m}}} \int_{i}^{t+T_{i}}\left(y_{i}(t)-\hat{m}_{y_{i}}(t)\right)^{2} d t\left|; \quad \hat{\sigma}_{y_{i}}(t)=\right| \sqrt{\frac{1}{T_{k c m}}} \int_{i}^{t+T_{t}}\left(d\left(v_{i}(t)-\hat{m}_{h_{i}}(t)\right) / d t\right)^{2} d t \mid .
$$

где $T_{\kappa c m}^{m \prime}$ и $T_{\kappa c m}$-интервалы квазистационарности $\hat{m}_{y_{i}}(t)$ и $\hat{m}_{i_{i}}(t), \hat{\sigma}_{y_{i}}(t)$ и $\hat{\sigma}_{i_{i}}(t)$.

Работа подсистемы управления конечной влажностью зерна $W_{3 K}$ не требует дополнительных комментариев, т.к. она имеет автономный, выделенный только для этого ресурс управления изменение производительности зерносушилки $G_{3}$. Управление температурами СА и зерна ведется в условиях совместного использования ресурсов управления - расхода топлива $G_{7}$ и подсоса атмосферного воздуха $G_{B}$, что гребуег пояснений

Регуляторы температуры СА и зерна (в соответствуюших зонах) включены каскадно через повторители с регулируемым верхним ограничением (ПРО). При этом значения этих ограничений устанавливаются на уровнях $T_{C A 1}^{\text {и }} T_{C A 2}^{\text {зы }}$. Управляюшие воздействия главных регуляторов (регуляторов температур зерна $T_{31}$ и $T_{32}$ ), когда их контура регулирования замкнуты, представляют собой такие заданные значения $T_{C 41}^{3 d}$ и $T_{C A 2}^{3 d 2}$ вспомогательным регуляторам, при которых их регулируемые переменные $T_{n}$ и $T_{32}$ будут равны своим заданным значениям, т.е. $T_{31} \approx T_{31}^{3 d d+}$ и $T_{32} \approx T_{32}^{3 d d+}$

Когда значения $T_{C A 1}^{30 i}$ и $T_{C A 2}^{30 ;}$ меньше своих предельно допустимых значений $T_{C A 1}^{30+}$ и $T_{C A 2}^{30 d+}$, т.е. $T_{C A l}^{3 d ;}<$ $T_{C A 1}^{\text {idd+ }}$ и $T_{C A 2}^{\text {id }}<T_{C A 2}^{\text {idd }}$, то регуляторы температур СА будут обеспечивать их подлержание равными $T_{C A 1} \approx T_{C A l}^{\text {sd }}$ и $T_{C A 2} \approx T_{C A 2}^{u d}$. Такая ситуация возникает, если вероятности нарушения допустимых границ температурами СА, т.е. вероятности возникновения событий $S_{C A 1} \rightarrow T_{C A 1}>T_{C A 1}^{p+}$ и $S_{C A 2} \rightarrow T_{C A 2}>T_{C A 2}^{z+}$ меньше, чем вероятности нарушения допустимых границ температурами зерна, т.е. вероятности возникновения событий $S_{31} \rightarrow T_{31}>T_{31}^{T^{*}}$ и $S_{32} \rightarrow T_{32}>T_{32}^{2 F}$ Следует отметить, что при этом каскадные структуры регуляторов работают по своей классической схеме.

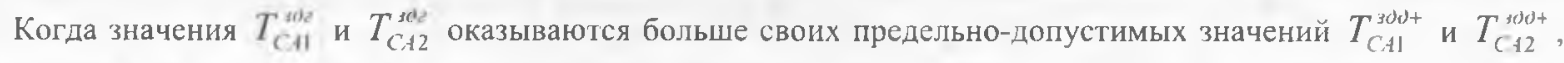
т.е. $T_{C A 1}^{3 d z}>T_{C A 1}^{3 d d+}$ и $T_{C A 2}^{3 d z}>T_{C A 2}^{3 d d+}$, то на выходе повторителей с регулируемыми ограничениями будут устанавливаться значения переменных, равные вычисленным ограничениям на заданные значения регуляторам температур СА, т.е. значения $T_{C A 1}^{30 d+}$ и $T_{C A 2}^{\text {зоd }}$ Именно в окрестностях этих значений и будут подлерживаться температуры СА, а температуры зерна будут изменяться «произвольно», т.к. в данной ситуации вероятности нарушения допустимых границ температурами СА, т.е. вероятности возникновения событий $S_{C A 1} \rightarrow T_{(' A 1}>$ $T_{C A 1}^{i p+}, S_{C A 2} \rightarrow T_{C A 2}>T_{C A 2}^{\iota p+}$ болыше, чем вероятности нарушения допустимых границ температурами зерна, т е вероятности возникновения событий $S_{31} \rightarrow T_{31}>T_{31}^{p i}$ и $S_{32} \rightarrow T_{32}>T_{32}^{\text {;p }}$. Следует отметить, что при этом каскадные структуры регуляторов перестают работать, т.к. главные обратные связи через регуляторы температур зерна размыкаются из-за работы ограничителей. Для подготовки к замыканию этих контуров, точнее для обеспечения «безударности» этого замыкания, необходимо значения интегрирующих составляющих принудительно устанавливать равными $T_{C A 1}^{3 d d+}$ и $T_{C A 2}^{30 d+}$, непрерывно отслеживая их

Синтез и анализ представленной системы проводился на имитационной, полнофункциональной модели с распределенными параметрами [2], которая с высокой степенью адекватности воспроизводит во времени процесс работы зерносушилки ДСП-32от. Для оценки эффективности разработанной СГУ был проведен ряд экспериментов, включаюший сравнительный анализ ее работы с работой штатной системы управления, в которой температуры СА по зонам стабилизируются регуляторами на предельно допустимых уровнях. Сравнительный анализ проводился при следующих условиях: $T_{\text {ral }}^{2 p+}=130^{\circ} \mathrm{C}, T_{a 2}^{2 p+}=150{ }^{\circ} \mathrm{C}, T_{\mathrm{rl}}^{p^{p+}}=T_{32}^{2 p^{+}}=50$ ${ }^{\circ} \mathrm{C}, W_{s k}^{2 p+}=14,5 \%, W_{y k}^{p-}=13,5 \%$. Параметры зерна, идушего на сушку, окружающего воздуха и топлива изменялись в соответствие с рисунком 2.

Фрагменты моделирования штатной системы управления для всех трех вариантов изменения параметров зерна, идущего на сушку, окружающего воздуха и топлива, без вмешательства оператора зерносушилки в процесс управления, приведены на рис. 3

Важно обратить внимание, что для этого случая, в ходе специальных машинных экспериментов, для системы были подобраны такие заданные значения температур СА $T_{c a 1}$ и $T_{c a 2}$, чтобы при неизменных средних значениях параметров зерна выполнялись бы все заданные регламентом сушки ограничения. Их пришлось 
установить значительно ниже допустимых: $T_{\text {ral }}=100^{\circ} \mathrm{C}$, а $T_{c a 2}=120^{\circ} \mathrm{C}$. Как видно из рисунка, шттатная система управления дает достаточно хоропие результаты только в случае, если среднее значение влажности $W_{\text {sн }}$ зерна, идуцего на сушку, остается неизменным (вариант «а»). В других случаях (варианты «б» и «в») обеспечить соблюдение регламентов не удается. На рис. 4 приведен фрагмент моделирования СІУ для варианта “в» нзменения начальной влажности и температуры зерна. Следует обратить внимание на то, что в СГУ, для выполнения требований гарантированного соблюдения установленных ограничений на температуры нагрева зерна, заданные значения температур $T_{\text {cal }}$ и $T_{\text {เа2 }}$ СА заранее не рассчитываются. Они определяются автоматически в ходе управления процессом сушки и сушественно изменяются, периодически приближаясь к своим предельно допустимым значениям $T_{\text {cat }}^{2 p+}$ и $T_{c a 2}^{2 p+}$, но не нарушая их.

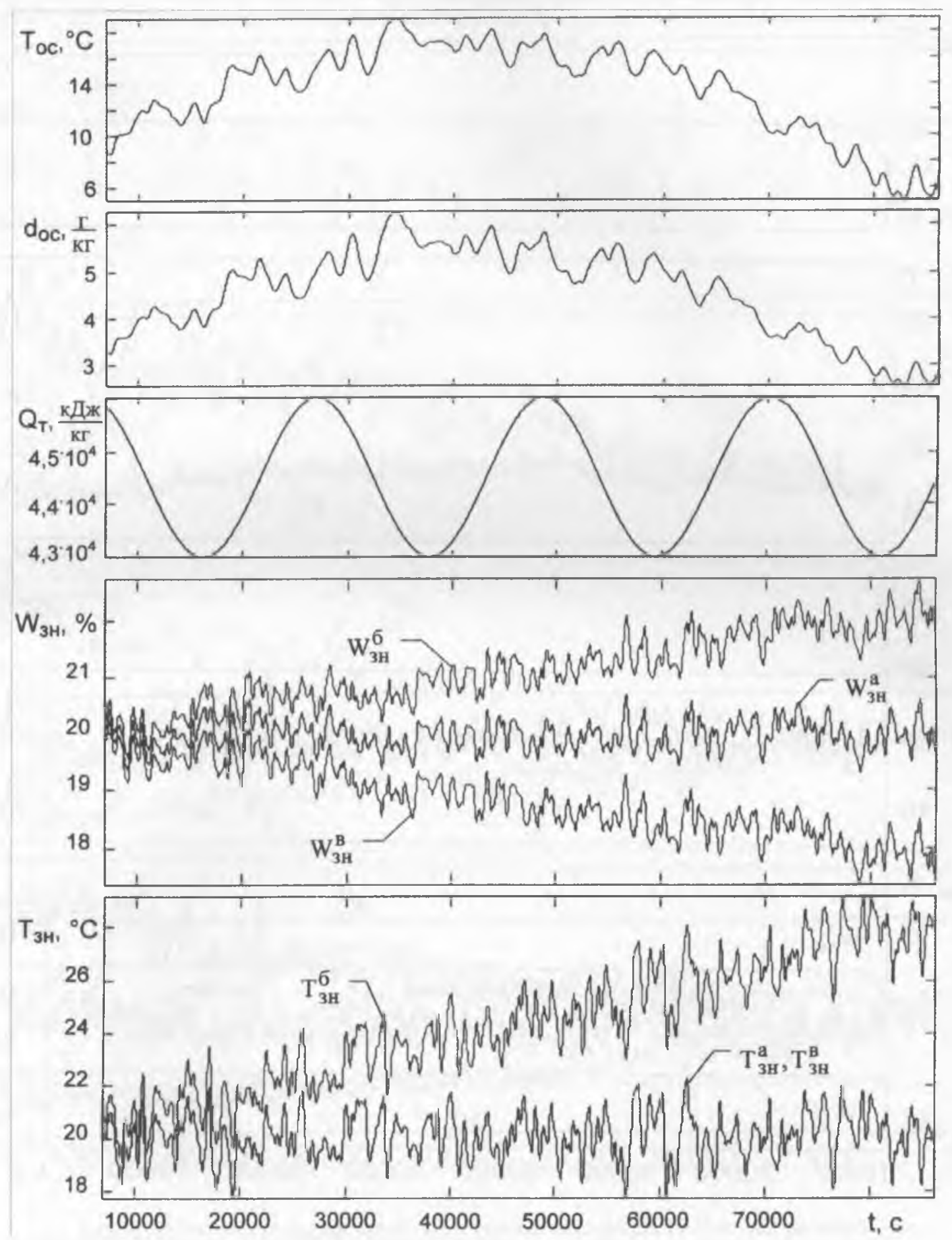

Рис. 2 - Динамика изменения параметров, определяющих условия ведения процесса сушки

$\left(T_{e c}, d_{e c}\right.$ - температура и влагосодержание окружающего воздуха; $\boldsymbol{W}_{3 \kappa}, T_{{ }_{\jmath}}$ - влажность и температура зерна, идущего на сушку; $Q_{m}$ - удельная теплота сгорания топлива) 

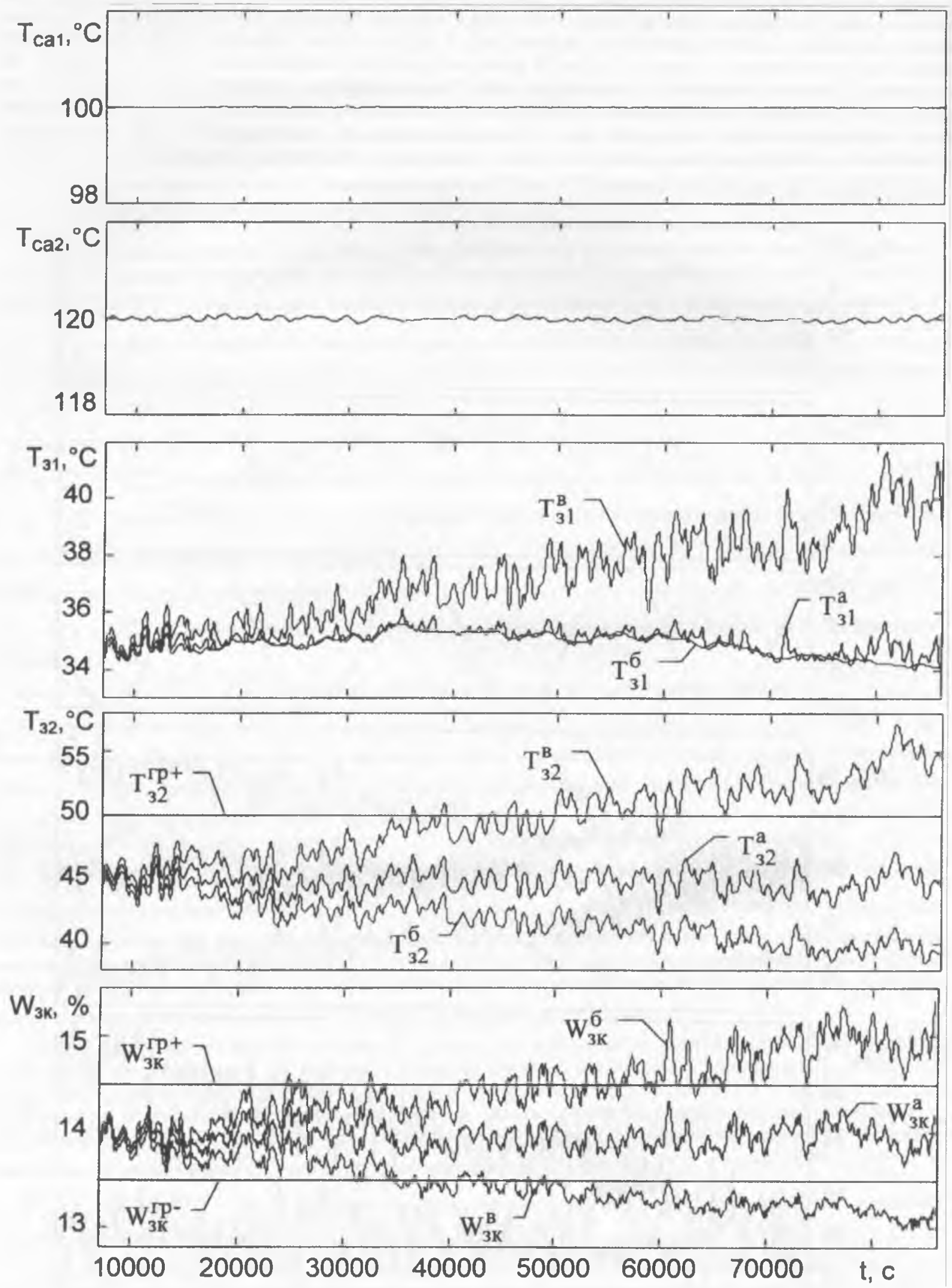

Рис. 3 - Фрагменты моделирования работы штатной САУ зерносушилкой ДСП-32о1 для трех различных вариантов изменения исходных параметров зерна 

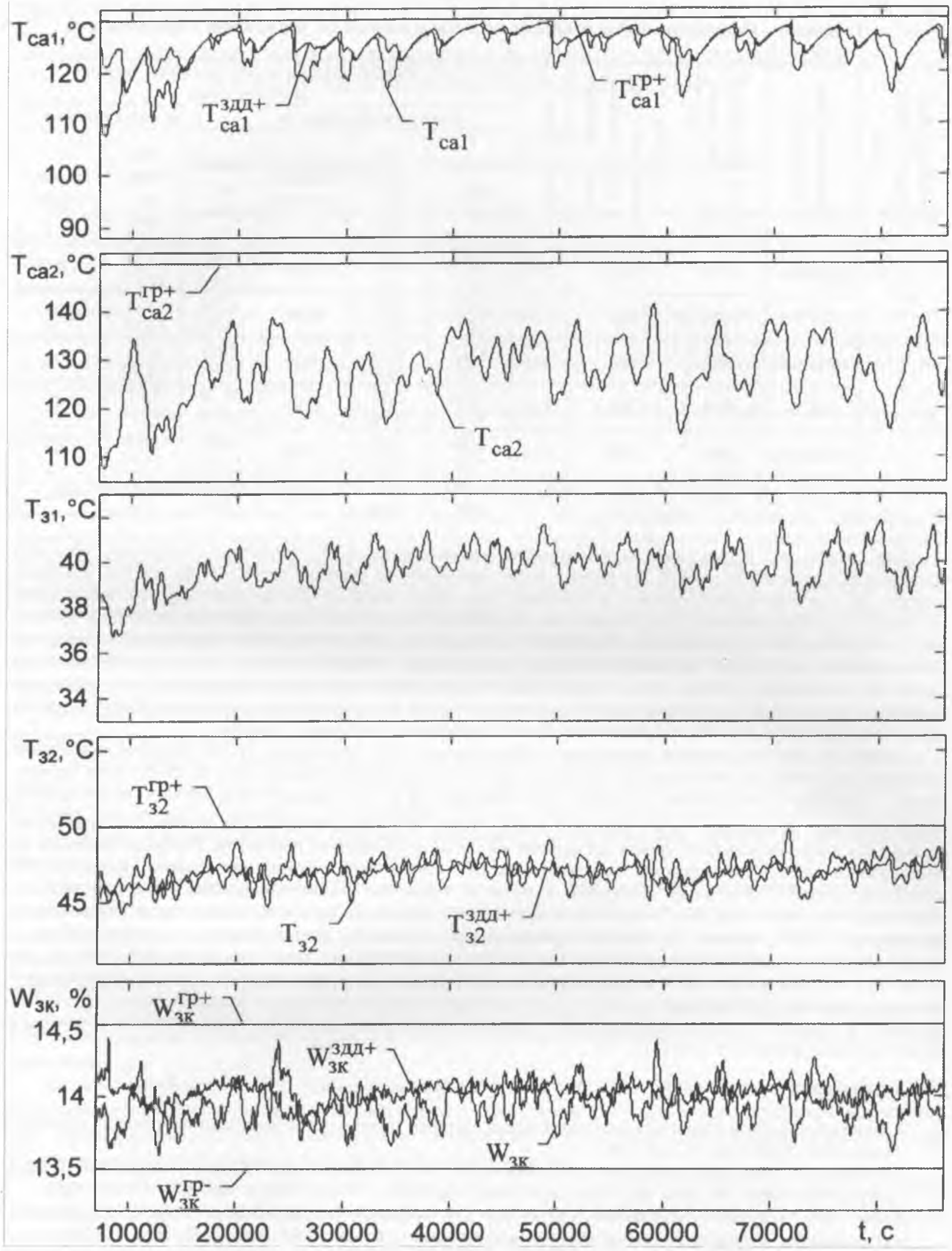

Рис. 4 - Фрагмент моделирования СГУ зерносушилкой ДСІІ-32от для варианта «В» изменения начальных значений параметров зерна (см. рис. 2)

Показатели эффективности ведения процесса для различных вариантов управления сведены в габлицу 1. 
Таблица I - Показатели эффективности различных вариантов управления зерносушилкой

\begin{tabular}{|c|c|c|c|c|c|c|c|c|c|c|}
\hline \multirow{3}{*}{ 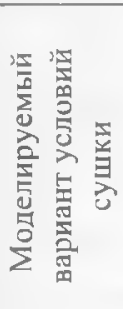 } & \multirow{3}{*}{ 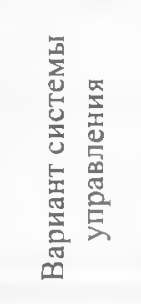 } & \multirow{3}{*}{ 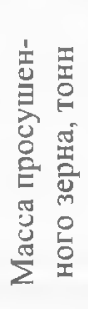 } & \multirow{3}{*}{ 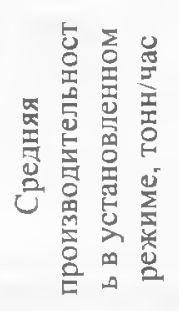 } & \multirow{3}{*}{ 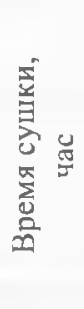 } & \multicolumn{6}{|c|}{$\begin{array}{c}\text { Затраты топлива и электрической энергии } \\
\text { на сушку зерна } \\
\end{array}$} \\
\hline & & & & & \multicolumn{3}{|c|}{ Электрическая энергия } & \multicolumn{3}{|c|}{ Дизельное топливо } \\
\hline & & & & & $\begin{array}{c}\text { Общие } \\
\frac{\mathrm{kBT}^{*}}{\Gamma \mathrm{pH} .}\end{array}$ & $\begin{array}{c}\mathrm{Ha} \\
\text { гонну } \\
\frac{\mathrm{KBT} 4}{\Gamma \mathrm{q}} .\end{array}$ & $\begin{array}{l}\text { Относи- } \\
\text { тельные }\end{array}$ & $\begin{array}{c}\text { Общие } \\
\underline{\kappa \Gamma} \\
г p н .\end{array}$ & $\begin{array}{c}\mathrm{Ha} \\
\text { тонну } \\
\underline{\mathrm{\kappa} \Gamma} \\
\ulcorner\mathrm{pH} .\end{array}$ & $\begin{array}{l}\text { Относи- } \\
\text { тельные }\end{array}$ \\
\hline \multirow[b]{2}{*}{ a } & IIIтатная & 1000 & 35,0 & 29.8 & $\frac{3719}{595}$ & $\frac{3.72}{0,6}$ & 1,00 & $\frac{7590}{15190}$ & $\frac{7.59}{15,19}$ & 1,00 \\
\hline & Сгу & 1000 & 42,1 & 25,4 & $\frac{3171}{507}$ & $\frac{3.17}{0,51}$ & 0,85 & $\frac{7330}{14660}$ & $\frac{7,33}{14,66}$ & 0,97 \\
\hline \multirow{2}{*}{6} & IIIтатная & 1000 & 31,5 & 33,0 & $\frac{4121}{659}$ & $\frac{4.12}{0,66}$ & 1,00 & $\frac{8450}{16900}$ & $\frac{8,45}{16,90}$ & 1,00 \\
\hline & $\mathrm{CI} y$ & 1000 & 38,0 & 27,9 & $\frac{3490}{558}$ & $\frac{3.49}{0,56}$ & 0,85 & $\frac{8170}{16340}$ & $\frac{8.17}{16,34}$ & 0,97 \\
\hline & IIIтатная & 1000 & 39,8 & 26,3 & $\frac{3286}{526}$ & $\frac{3.29}{0,53}$ & 1,00 & $\frac{6680}{13350}$ & $\frac{6,68}{13,35}$ & 1,00 \\
\hline & СГУ & 1000 & 49,2 & 21,9 & $\frac{2738}{438}$ & $\frac{2.74}{0,44}$ & 0,83 & $\frac{6200}{12400}$ & $\frac{6,20}{12,40}$ & 0,93 \\
\hline
\end{tabular}

Как видно из результатов моделирования использование СГУ позволило увеличить среднюю производительность на $20-24 \%$, уменышить общее время работы сушилки на $15-17 \%$, снизить удельные затраты на электрическую энергию и топливо. При этом система обеспечивает строгое соблюдение регламентов ведения процесса. Актуальность использования этой системы возрастает в период уборки урожая, когда дополнительно к требованиям соблюдения регламентов, снижения удельных энергозатрат добавляется необходимость увеличения производительности зерносушилок. Следует отметить, что в модели процесса сушки не учитывались потери тепла в окружающую среду через поверхности конструкций всех элементов сушилки. Это означает, что снижение затрат топлива на сушку при повышении производительности сушилки фактически будет больше, чем получено методом моделирования и отражено в таблице.

Одним из важных резервов повышения эффективности СГУ процессом сушки является повышение динамической точности стабилизации влажности зерна на выходе из сушилки. Это позволит вести процесс

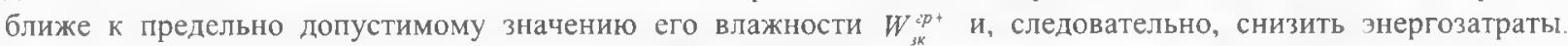
Инструментами повышения этой точности может стать развитие системы управления в направлении повышения интеллектуального уровня алгоритмов контроля и управления процессом. Наиболее важными на сегодняшний день, по-видимому, следует считать следующие направления такого развития: 1) совершенствование алгоритмов измерения влажности зерна на основе применения тестовых методов измерения, что может повысить точность и стабильность существуюших технических средств измерения; 2) реализация в САУ, помимо принципов гарантирования, принципов инвариантности, в данном случае инвариантности влажности зерна на выходе из сушилки от значения его влажности на входе в сушилку. Он потребует интегрирования в САУ математической модели процесса и ее параллельного с процессом решения в реальном времени; 3) применение в САУ качестве алгоритмов регулирования специальных. ориентированных на работу в условиях больших запаздываний и вариаций параметров, которыми обладают каналы управления объектов технологического типа [3].

\section{Литература}

1. Хобин В.А. Системы гарантирующего управления: научные основы // Автоматизація виробничих процесів. - Київ, 2004. - № 2 (19). - С. 62 - 69.

2. Степанов М.Т. Имитационная математическая модель процесса сушки зерна. // Нове в технології зберігання та переробки зерна:- 36. наук. пр. / Одес. держ. акад. харч. техн. Одеса, 2002. Вип. 24. С. 380384

3. Хобин В.А. Регулятор переменной структуры для объектов техологического типа // Автоматика. Автоматизация. Электротехнические системы и комплексы. - Херсон, 2004 - № 1 (13). - С. 190 - 196. 